\title{
GT2020-16059
}

\section{EXPLORING TOPOLOGY OPTIMISATION OF HIGH PRESSURE TURBINE BLADE TIPS}

\author{
Luka Vincekovic* \\ Alistair John \\ Ning Qin \\ Department of Mechanical Engineering \\ University of Sheffield \\ Sheffield, United Kingdom \\ Ivincekovic1@sheffield.ac.uk
}

\author{
Shahrokh Shahpar \\ Innovation Hub - Central Technology - Future Methods \\ Rolls-Royce plc \\ Derby, United Kingdom
}

\begin{abstract}
This work presents the aerodynamic topology optimisation of high pressure turbine rotor blade tips. Before carrying out the topology optimisation on the blade tip, some initial tip design studies were carried out. The winglet shape was optimised using two different design space setups and parameter limits. The optimum winglet design features the largest overhangs and in the case of unconstrained optimisation proved to have $1.40 \%$ greater aerodynamic efficiency. Secondly, a radial basis function based parametrisation was set up to allow the creation of single squealer line using the flat tip blade as a baseline geometry. The optimum case proved to increase efficiency $0.46 \%$ compared to the flat tip. After that, a combination of winglet and topology free squealer tips was investigated for topology optimisation. The winglet tip was parametrized as in the winglet only optimisation cases and topology free squealer walls were created using mapping of radial basis function surfaces of different complexities. It is shown that by combining both winglet and novel squealer topology optimisation, better designs of different topologies can be produced.
\end{abstract}

\section{NOMENCLATURE} Symbols

$\begin{array}{ll}\text { s } & \text { entropy } \\ \text { C } & \text { specific heat capacity }\end{array}$

$\begin{array}{ll}\gamma & \text { specific heat capacity ratio } \\ \mathrm{M} & \text { Mach number } \\ \dot{m} & \text { mass flow } \\ \mathrm{V} & \text { velocity } \\ \Theta & \text { mismatch angle } \\ \mathrm{p} & \text { pressure } \\ \mathrm{T} & \text { temperature }\end{array}$

$\begin{array}{ll}\text { Subscripts } \\ \text { mix } & \text { mixing } \\ \mathrm{p} & \text { at constant pressure } \\ \text { leak } & \text { leakage } \\ 0 & \text { total } \\ \text { st } & \text { static } \\ \text { is } & \text { isentropic }\end{array}$

\begin{tabular}{ll}
\multicolumn{2}{l}{ Abbreviations } \\
PS & Pressure Side \\
SS & Suction Side \\
LE & Leading Edge \\
TE & Trailing Edge \\
SOPHY & SOft-Padram-HYdra \\
RANS & Reynolds-Averaged Navier Stokes \\
CFD & Computational Fluid Fynamics \\
PADRAM & PArametric Design and RApid Meshing \\
MAM & Multipoint Approximation Method
\end{tabular}




$\begin{array}{ll}\text { SOFT } & \text { Smart Optimisation For Turbomachinery } \\ \text { RBF } & \text { Radial Basis Function } \\ \text { OTL } & \text { Over Tip Leakage } \\ \text { DOE } & \begin{array}{l}\text { Design Of Experiments } \\ \text { ARMOGA }\end{array} \\ \begin{array}{l}\text { Adaptive Range Multi-Objective Genetic Algo- } \\ \text { rithm }\end{array}\end{array}$

\section{INTRODUCTION}

Unshrouded high pressure turbine rotors have been recently introduced in large jet engines for commercial use. Compared to the shrouded ones, unshrouded rotors are superior in the terms of weight and cooling delivery requirements. However, because of the absence of the shroud, new ways of controlling the tip leakage flow have to be found.

Turbine tip leakage is the main source of efficiency loss in turbines of jet engines and the primary factor when it comes to the periodic maintenance demands. The ideal turbine blade would not have any clearance between the blade tip and the casing therefore allowing no leakage of working fluid over the tip. Leakage of the working fluid occurs over the blade tip, from pressure side to the suction side of the blade and according to Denton [1], is associated with up to $30 \%$ of the total aerodynamic loss in the stage of a modern high pressure turbine. Efficiency loss due to the leakage over the blade tip can roughly be divided into two mechanisms. Firstly, mass flow that leaks over the tip does not fully contribute by doing work on the turbine blade and secondly, leakage flow exiting over the blade tip increases viscous effect due to strong shear with the primary flow which leads to entropy generation in the adjacent turbine channel. Entropy generation due to mixing of the main passage flow and leakage flow is summarized in Eqn. (1) by Denton [1].

$$
\Delta s_{\text {mix }}=C_{p}(\gamma-1) \frac{\dot{m}_{\text {leak }}}{\dot{m}_{\text {passage }}} M_{S S}^{2}\left(1-\frac{V_{\text {leak }}}{V_{S S}} \cos (\Theta)\right)
$$

According to Denton [1], mixing losses $\Delta s_{\text {mix }}$ increase with leakage mass flow $\left(\dot{m}_{\text {leak }}\right)$, freestream Mach number $(M)$ and the mismatch angle $(\Theta)$ between the freestream and leakage flow. Therefore, new turbine tip designs aim to reduce leakage mass flow and inject it into the regions of lower Mach number with smaller mismatch angle towards the freestream.

As turbine tips are subjected to high heat load, it is common practice when performing an optimisation process to examine all the designs in terms of both aerodynamic efficiency and heat load. However, as this paper emphasizes exploring topology optimisation, finding a robust routine for aerodynamic topology optimisation first, is in scope.

\section{PREVIOUS STUDY ON BLADE TIP AERODYNAMICS}

In order to design a rotor blade tip of enhanced aerodynamic performance, it is crucial to correctly identify all the flow phenomena in the passage of the turbine stage. Sieverding [2] explained interaction between passage and horseshoe vortices, their effect on endwall boundary layer and dissipation loss.

When it comes to the tip shapes, various configurations of squealer and winglet designs have been published. Tip leakage over the flat tip blade has been described by Coull and Atkins [3]. Squealer tips featuring various wall openings were examined by Vass and Arts [4]. The authors state that the full perimeter squealer features the lowest kinetic energy losses, compared to the partial suction and pressure side squealer. Simple and optimised squealer tips leakage flow has been described in details by Pátý et al. [5] experimentally and numerically. Li et al. [6] examined the effect of the squealer rim height and width for a full perimeter squealer. The optimum squealer rim height was found to be 3 times the tip gap. Further increase of the squealer wall height was found to have an adverse effect on preventing the leakage flow. The optimum squealer rim width was found to be when the width was the same as the tip gap, the thinnest rim tested. Du et al. [7] examined one flat and five squealer designs where cavities inside squealers were created by adding the rims perpendicular to the blade camber line. It was found that adding rims perpendicular to the blade camber line worsens the performance of a simple squealer.

Tip leakage loss has been investigated by Schabowski and Hodson [8] experimentally using a turbine cascade and numerically for the different squealer and winglet tips and their combinations. The authors state three major methods of the tip leakage prevention. First is the reduction of the pressure difference using a winglet tip with overhangs. Second is using two squealers with leading and trailing edge openings which allow splitting the total leakage flow into two parts, one leading over the suction side rim and the other through the opening at the trailing edge. The third method is using rims thinner than the tip gap to prevent flow reattaching to their top surface.

Coull et al. [9] performed an aerothermal parametric study of winglet tips. They investigated winglet tips with full and partial overhangs around the tip edge and found out that all winglet designs outperformed the flat tip in the terms of aerodynamic efficiency. The full perimeter winglet was found to be the most efficient but also the highest heat load. The authors state that winglet efficiency benefit results from overhangs which allow for the leakage flow to be injected further from the blade, into the region of higher pressure which reduces mixing losses. Coull et al. [10] investigated the performance of cavity winglets and compared them to the flat tip and conventional winglet and squealer designs. They state that adding the cavity to the winglet increases the efficiency by causing low pressure zones which results in more flow ingestion around the leading edge. The highest efficiency is achieved for a cavity-winglet with full perimeter 
overhang. Caloni et al. [11] did an aerothermal optimisation of squealer tips with and without overhangs. The authors showed that significant efficiency benefit with almost constant or reduced heat load can be achieved by combining small overhangs and openings at LE and TE.

De Maesschlack et al. [12] did a shape optimisation of the tip geometry on 2D planes at three different axial positions. The study was performed for various tip width-to-gap ratios and loadings ranging from the subsonic to supersonic flow conditions. The authors conclude that the tip geometries with a superior performance in the subsonic conditions are not optimal in the supersonic conditions because of different loss production mechanisms. De Maesschlack et al. [13] also performed a multi-objective shape optimisation for carved (contoured) and squealer-like designs using two different tip parametrization approaches. Results show that the flat tip outperforms the carved shape when considering aerodynamic efficiency only. However, carved designs decrease the heat transfer level. Comparing the squealer and carved design, authors state that squealer tips are superior to carved designs, offering larger reduction in the heat load for the price of smaller aerodynamic efficiency drop. Andreoli et al. [14] did a multi-objective optimisation of turbine tips with film cooling. They parametrized geometry using mapping of the Bezier surface in non-dimensional plane which represented the blade tip. The authors produced new squealer-like designs which are superior in both aerodynamic efficiency and lower heat load.

\section{METHODOLOGY}

As shown by Caloni et al. [11], blade tips which combine common designs like winglet and squealer shapes with different openings can bring efficiency benefit without increase in heat load. Andreoli et al. [14] showed that creating a topology free optimisation of squealer tips has a great potential in exploring different squealer topologies in single optimisation workflow. Therefore, in this work, different optimisations are carried out, gradually exploring a design space to create the novel topology free turbine tip optimisation. In the following sections, the geometry parametrization and creation methods are presented first. After geometry creation, numerical modelling is explained with model validation and verification.

\subsection{Geometry parametrization}

3.1.1 Winglet The winglet geometries were parametrized and generated using PADRAM [15], Rolls-Royce in-house tool for parametrization and geometry and mesh generation. Starting from the datum rotor blade presented in Fig. 3 as a basis, this process is illustrated in Fig. 1. The winglet tip outline is defined at different control points around the datum tip (black line in Fig. 1a). Overhangs which create the winglet tip are defined with five points around the blade perimeter, one at the leading edge, one at the suction and the pressure side and two at the trailing edge. The position of each of the leading edge, suction side and pressure side points is a parameter allowing movement in the streamwise direction, with trailing edge points fixed. With overhang point positions defined at the datum outline, overhang values, which are also parameters, are expressed as a percentage of axial chord. Overhang points are then moved from their positions on datum outline in normal direction for overhang value. After that, with winglet overhang control points fully defined, the outline of the winglet tip (red line in Fig. 1a) is created by interpolating between the overhangs points along the datum outline. With the winglet tip outline created, the tip is extruded for a straight length in the radial direction toward the hub. Sharp edges are kept around the tip edge. The straight extrusion length (shown in Fig. 1b) is defined as a parameter at each overhang point separately and interpolated between. After that, the blending angle, parametrized at each point, determines angle under which overhang merges with the datum blade.

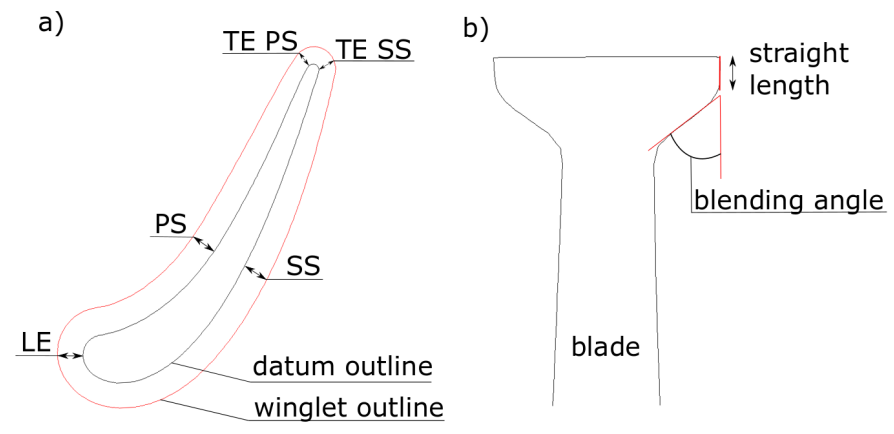

FIGURE 1. WINGLET PARAMETRIZATION

3.1.2 Topology free squealer Squealer tips were parametrized and created using mimic [16], software for geometry manipulation from OPTIMAD [17]. Mimic uses a radial basis function to create a description of squealer lines in a nondimensional space which are then mapped on the top of the datum blade. The radial basis function is created by defining a number and the height value of points through which it is then interpolated.

An example of creating a topology free squealer tip using a winglet tip as a basis is shown in Fig. 2. An arbitrary set of points is defined in the non-dimensional space as shown in Fig. 2a. Points have fixed $x-y$ position and are allowed to move in the z-direction. Interpolating through these points, an RBF surface is created. With RBF surface defined and created, the 
description of squealer lines in the non-dimensional space is obtained by cutting the RBF surface at zero height. Fig. $2 \mathrm{~b}$ presents the contour of RBF surface in the non-dimensional space viewed from above with sqeualer lines as a result of the RBF surface cut. These sqeualer lines are then mapped on the design space, in this case, the winglet tip (Fig. 2c). Finally, the squealer tip is produced by extruding the surface of the tip following the location of mapped squealer lines as shown in the Fig. 2 d.

As the number of points which form the RBF surface is arbitrary, design space complexity is totally flexible. Using this approach, squealer tip geometries that feature a different number and positions of lines and openings can be generated. Being allowed to define both a number, but also the mobility of the points, this free topology approach enables simultaneous exploration of new topologies and a design parametrization of potentially novel blade tip designs.

a)

b)
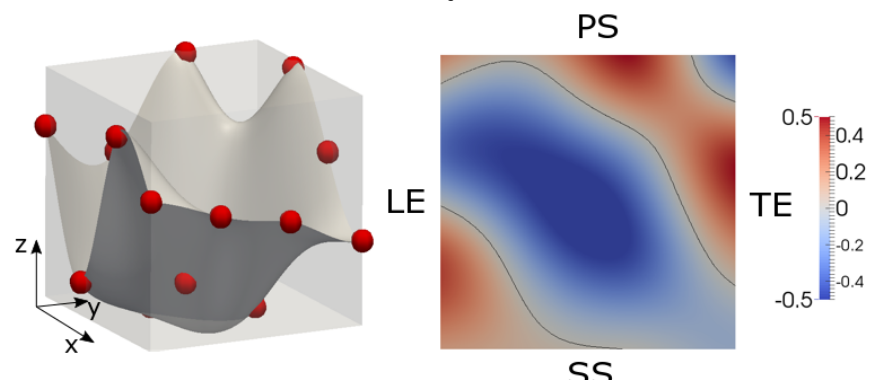

SS

C)

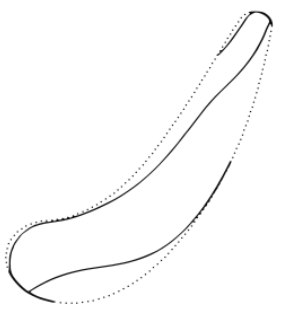

d)

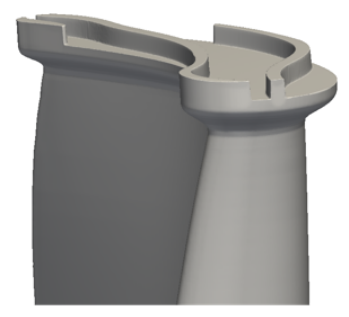

FIGURE 2. PARAMETRIZATION FOR TOPOLOGY OPTIMISATION

\subsection{Computational methods}

This work has been done on the MT-1 high pressure research turbine [18] presented in Fig. 3. The MT-1 turbine stage consists of 32 blades in the stator domain and 60 blades in the rotor domain, having one passage pitch of $11.25^{\circ}$ and $6^{\circ}$ respectively. Rotor tip gap used is $1.5 \%$ of blade span $(0.7 \mathrm{~mm})$ and constant for all the tested cases.

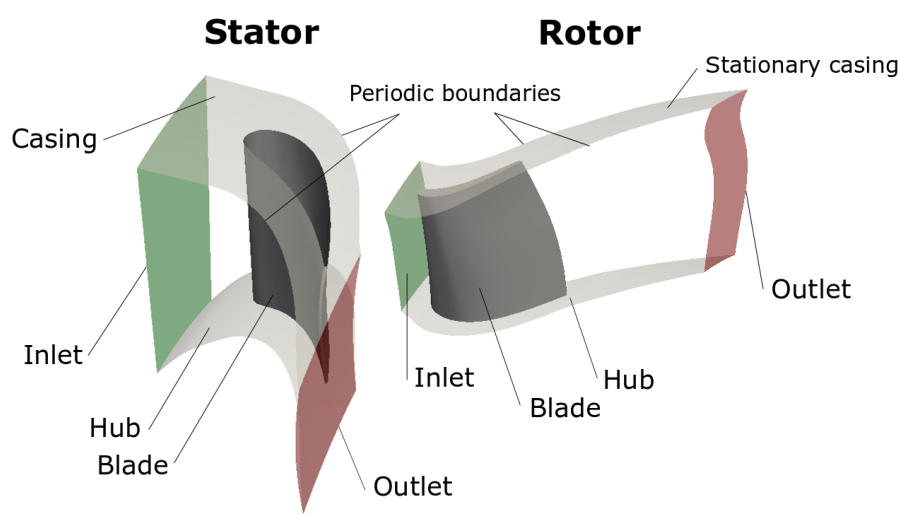

FIGURE 3. MT-1 TURBINE GEOMETRY

TABLE 1. BOUNDARY CONDITIONS

\begin{tabular}{cl}
\hline Boundary & Condition \\
\hline Stator inlet & $p_{0}, T_{0}$ \\
Stator outlet & mixing outflow \\
Rotor inlet & mixing inflow \\
Rotor outlet & $p_{s t}$ \\
Hubs, casings, blades & No-slip adiabatic walls \\
\hline
\end{tabular}

3.2.1 Numerical model Steady RANS simulations for a single turbine stage passage including one stator blade and one rotor blade were performed using Hydra, Rolls-Royce in-house CFD tool using the Spalart-Allmaras turbulence model [19]. The imposed boundary conditions are summarised in Tab. 1. At the stator inlet, uniform total pressure and total temperature were defined. The inlet flow direction was specified imposing whirl and pitch angles. Between the stator and rotor domain, a mixing plane was used, averaging the flow properties in the circumferential direction. The outlet of the rotor domain was defined with a static pressure distribution.

In all optimisations, aerodynamic stage efficiency was used as an objective, from now on referred to as efficiency. It has been calculated as presented in Eqn. (2).

$$
\eta=\frac{h_{0, \text { inlet }}-h_{0, \text { outlet }}}{h_{0, \text { inlet }}-h_{0, \text { is,outlet }}}
$$

3.2.2 Meshing and mesh convergence study The meshes used were generated using PADRAM and BOXERmesh

Copyright (c) 2020 by Rolls-Royce plc 
[20]. PADRAM, along with parametrizing and creating a geometry, is capable of the automatic multi-block structured mesh generation. A PADRAM mesh was used for the stator domain in all simulations, and for the rotor domain in the winglet optimisation cases. The PADRAM mesh convergence study was done for the case with datum flat tip blade and is presented in Fig. 4. The mesh size presented in Fig. 4 accounts for the both stator and rotor domain where mesh densities of both domains were increased equally. As an objective for evaluating different meshes, the aerodynamic efficiency was used. A mesh independent solution for PADRAM mesh was achieved for the 2 million cells mesh (red dot in Fig. 4) as with further refinement, aerodynamic efficiency has not changed by more than $0.05 \%$. This mesh is from now on referred to as the converged mesh. From almost 2 million cells in the whole stage, the stator mesh size was around 0.8 million cells.

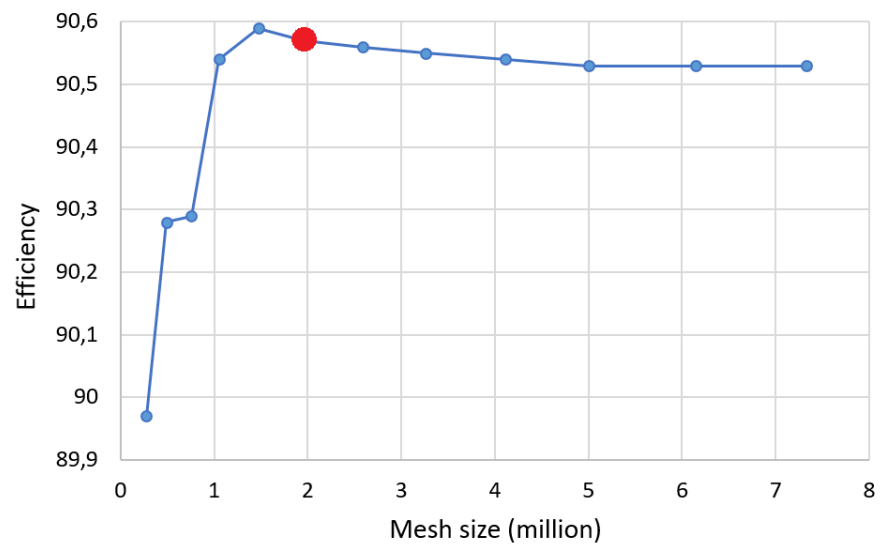

FIGURE 4. PADRAM MESH CONVERGENCE STUDY

In the optimisation cases where the geometry was parametrized using mimic creating squealer tips, rotor domain was meshed using BOXERmesh [20]. BOXERmesh is an octree based meshing tool which produces an unstructured hexdominant mesh with a boundary layer mesh. Additional refinements were applied in the tip region to ensure capturing the flow details. Unstructured meshing in this application has a few advantages over the structured meshing, easing the automation process and focusing mesh refinements in the flow sensitive areas. As the stator domain was not altered, the converged PADRAM mesh was used for the stator domain and kept constant. The advantage of using PADRAM mesh in the stator domain is its multi-block structure which adequately captures the flow features using fewer number of cells, saving on the simulation time and speeding up the optimisation process. The BOXERmesh convergence study was done by gradually refining the whole rotor domain mesh using the converged PADRAM mesh for the sta- tor domain which was kept constant. Results of BOXERmesh convergence study are presented in the Fig. 5 for a flat tip datum blade. The converged mesh had almost 9 million cells in the rotor domain (of which $1 / 3$ in the tip region) as after further refinement the aerodynamic efficiency changed negligibly.

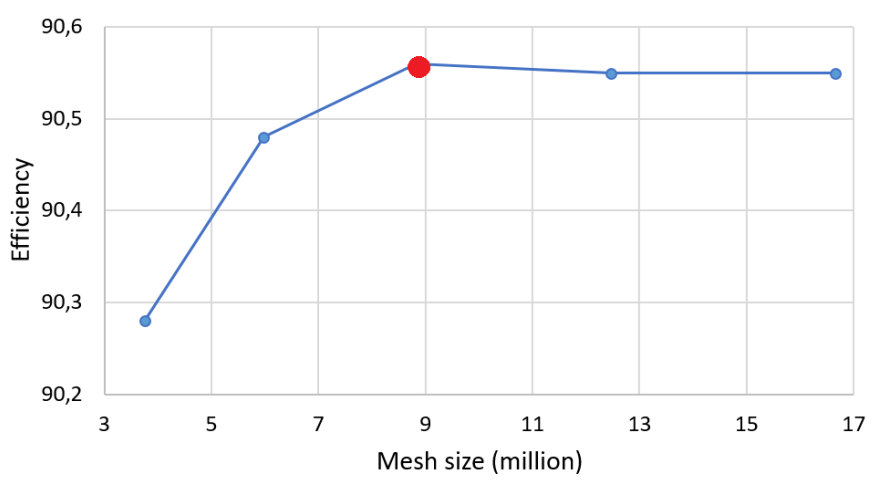

FIGURE 5. BOXER MESH CONVERGENCE STUDY

Details of PADRAM and BOXERmesh meshes are presented in the Fig. 6. PADRAM mesh (left) presents detail of the stator mesh at the joint of the stator blade and hub. BOXERmesh mesh (right) shows the level of refinement of the squealer tip region.

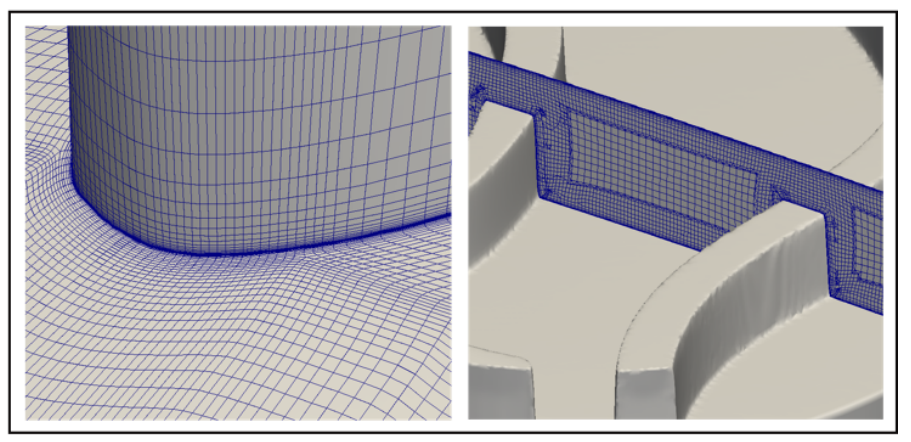

FIGURE 6. PADRAM AND BOXER MESH DETAIL

3.2.3 Experimental validation The numerical model was validated for datum flat tip blade against the experimental results from Beard et al. [21] in both the stator and rotor domains. Isentropic Mach number at 50\% of the blade span has been calculated using Eqn. (3) at the stator blade and compared to the experimental results as shown in Fig. 7. 


$$
M_{i s}=\sqrt{\left(\left(\frac{p_{0, \text { inlet }}}{p}\right)^{\frac{\gamma-1}{\gamma}}-1\right) \frac{2}{\gamma-1}}
$$

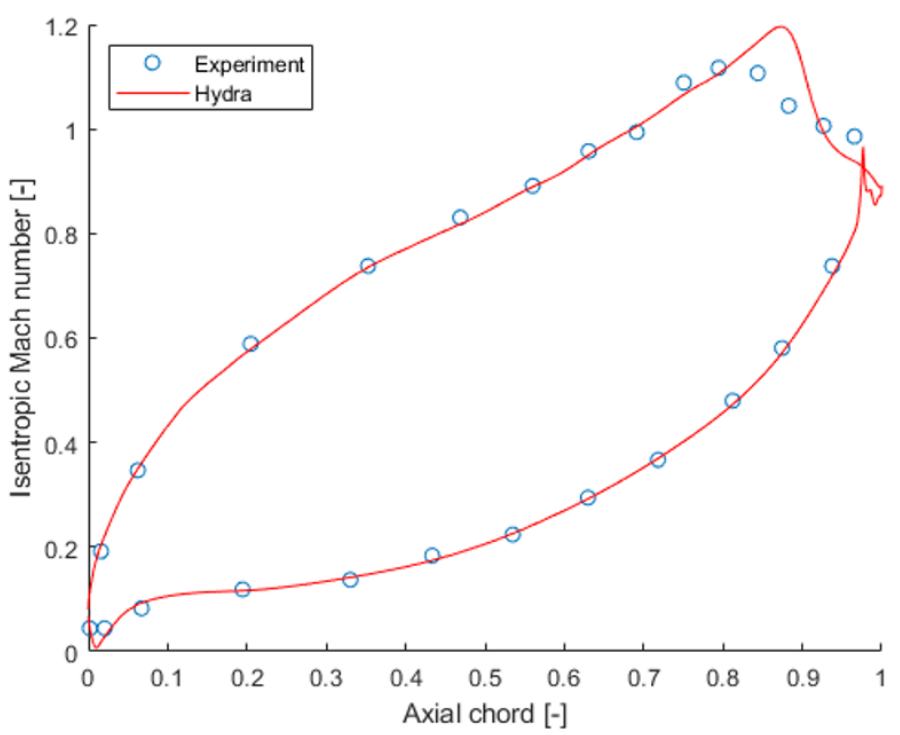

FIGURE 7. STATOR BLADE ISENTROPIC MACH NUMBER

The results show good agreement with experimental results at all regions apart from the aft region of the blade suction side. In this region numerical results predict a shock which results in region of increased isentropic Mach number when compared to the experimental data. This behaviour has been reported by few different studies [21,22]. Beard et al. [21] showed that the unsteady simulation averages out this phenomena.

For the rotor domain, the non-dimensional static pressure at $50 \%$ of the blade span is shown in the Fig. 8. Hydra slightly overpredicts the non-dimensional static pressure at all regions of the blade. These results are in agreement with the studies from Beard et al. [21], Salvadori et al. [22] and Simone et al. [23] using different codes. Their unsteady simulations have been found to produce similar deviation from the experimental data as the simulations done in this study. Different turbulence models have also been tested and produced a similar behaviour. Finer meshes have also been tested and compared against the experimental data, no better fit was found.

\section{OPTIMISATIONS AND RESULTS}

The optimisation process was carried out using SOPHY (SOFT-Padram-Hydra) [24], Rolls-Royce in-house system for

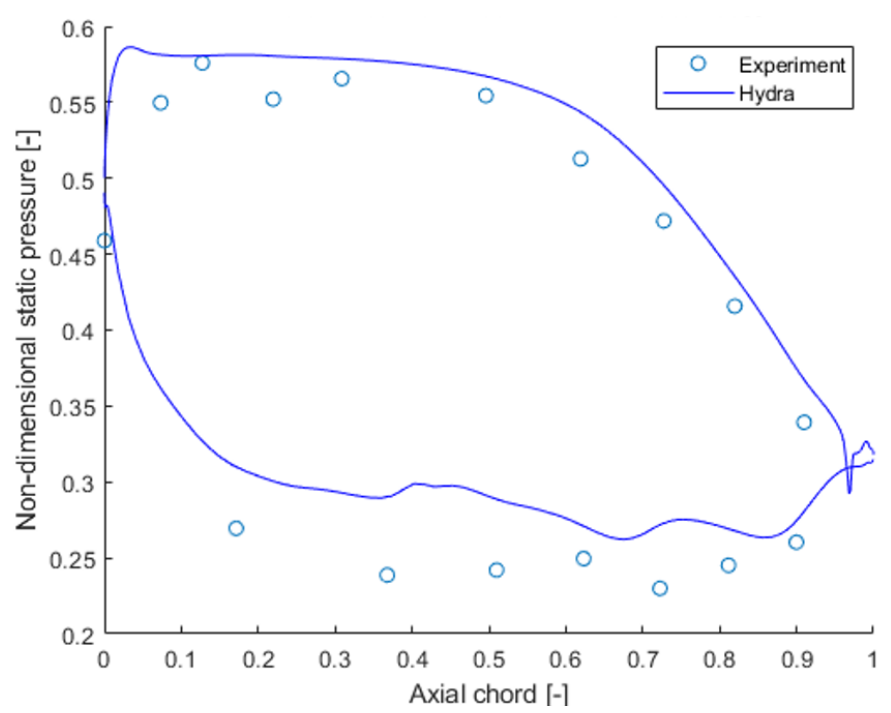

FIGURE 8. ROTOR BLADE NON-DIMENSIONAL STATIC PRESSURE

turbomachinery optimisations linking the processes of geometry parametrization and creation, flow solving and optimising design parameters. Different optimisers have been used, depending on the flexibility of design space, as described in further sections.

\subsection{Winglet optimisation}

The winglet tip was parametrized using 19 design parameters. After parametrization and creation of the geometry, the multi-block structured mesh was generated for the both rotor and stator domain using PADRAM. Aerodynamic stage efficiency was set up as the optimisation objective and Multipoint Approximation Method (MAM) [25, 26] available inside SOFT (Smart Optimisation For Turbomachinery) [27] optimiser was used. The whole optimisation routine is presented in Fig. 9.

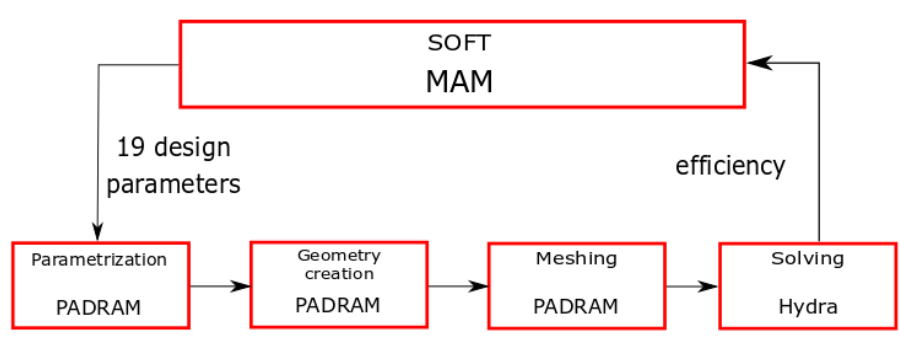

FIGURE 9. WINGLET OPTIMISATION ROUTINE

During winglet optimisation, almost 330 design iterations have been done. The optimised winglet tip outline is presented in Fig. 10 along with the datum tip outline. The optimised design

Copyright (c) 2020 by Rolls-Royce plc 
features full perimeter overhangs apart from the TE SS where the overhang is almost negligible. Interestingly, only a small overhang is present at the mid-PS of the blade where a significant amount of tip leakage flow is expected. In all other regions, maximum overhangs allowed are applied. Fig. 10 presents three winglet blade cross sections. All three cross sections show that the optimised winglet has no downwards extrusion of the tip (straight length in Fig. 1b) creating a sharp edge. Sharp edges are desired in tip leakage geometries as they cause separation bubbles which decrease effective tip gaps. However, it is expected that sharp winglet edges found in this study would be modified after considering heat load and thermal and mechanical stresses. Cross sections of the blade also present blending angles between overhangs and the datum blade. The blending angles applied are of almost maximum allowed value around the whole blade perimeter except mid-PS as there is almost no overhang. This led to the creation of winglet tip that is as thin as possible with full perimeter overhang (except mid-PS).
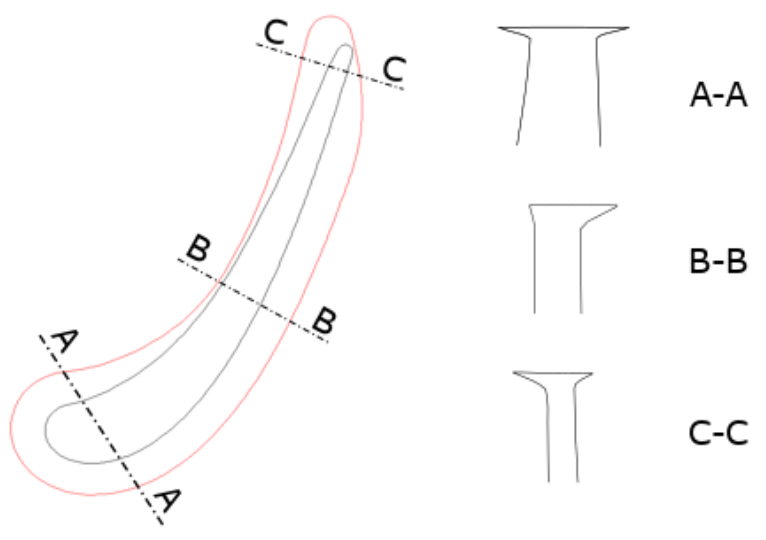

FIGURE 10. OPTIMISED WINGLET OUTLINE

\subsection{Single rim squealer optimisation}

The single rim squealer tip was parametrized and created using mimic, with 4 points controlling the RBF surface. The process of creating the initial optimisation design is shown Fig. 11. As in this optimisation only one squealer line was investigated, 2 points corresponding to the ends of PS were constrained as positive and 2 points corresponding to ends of SS as negative. This ensured the creation of only one squealer line which would stretch along the whole blade tip, connecting the LE and TE. As the points move independently in z-direction, the squealer line changes its position between PS and SS and its curvature.

In this optimisation, the squealer wall height and width were set up as parameters, ensuring always constant tip gap defined from squealer wall top surface. Along the $4 \mathrm{RBF}$ points, this a)

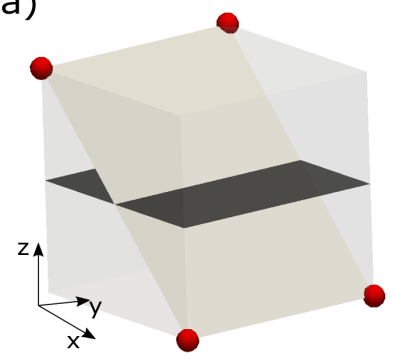

b)

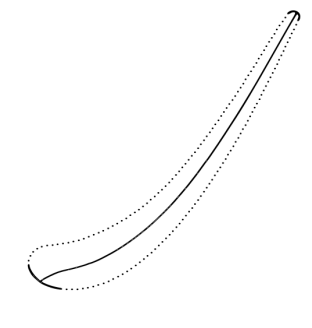

FIGURE 11. SINGLE RIM SQUEALER PARAMETRIZATION

optimisation had overall 6 parameters. MAM optimiser was used and whole optimisation routine is presented in Fig. 12.

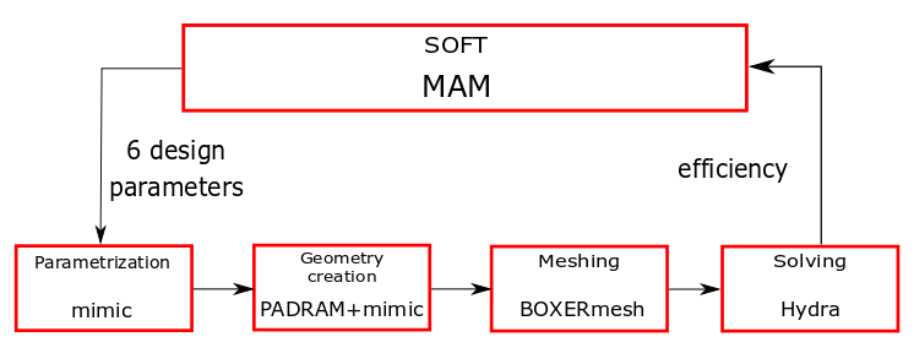

FIGURE 12. SINGLE RIM SQUEALER OPTIMISATION ROUTINE

The optimisation took around 60 iterations to converge and the optimised single line squealer is presented in Fig 13. The optimised design squealer line has moved to the pressure side of the blade except of the small region at the early PS where edge of the datum blade is visible. The reason for this was found to be closing of the leading edge around stagnation region. With constraints on the design parameters controlling the position of the RBF points, only one squealer line was allowed and the tradeoff between closing the PS and LE has to be found. As squealer the rim height and width were also part of the optimisation, the optimised design features a squealer rim width of $1.1 \%$ of axial chord length which is less than the tip gap. This is in agreement with recommendations from Schabowski and Hodson [8]. The height of the optimum squealer rim is $3.7 \%$ of axial chord length or around 1.5 tip gap.

\subsection{Winglet-topology free squealer optimisation}

The winglet-squealer blade was created using both PADRAM and mimic. However, PADRAM was used only to create a winglet tip blade as described in Fig. 1. The created winglet tip was then used as a baseline for creating the topology free squealer walls on top of it. During the whole optimisation 

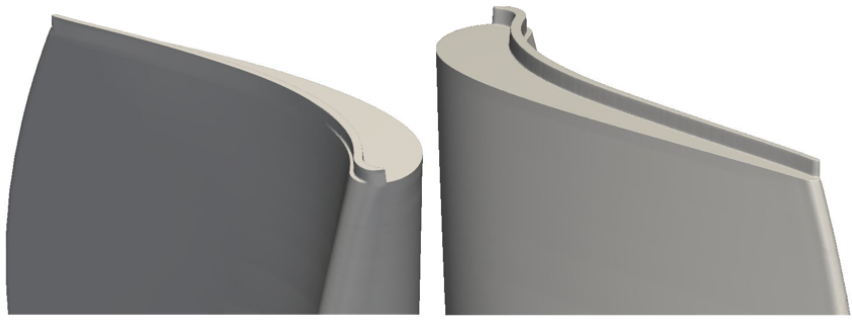

FIGURE 13. SINGLE RIM SQUEALER OPTIMISED GEOMETRY

process, the winglet tip parameters were kept constant producing always the same winglet baseline blade for all squealer designs and were not part of the optimisation process. Same as in the case of single rim squealer, constant tip gap was defined from squealer wall top surface. As the scope of this optimisation was to investigate novel squealer designs, 16 points were used to define the RBF surface, allowing complex squealer wall shapes (see Fig. 2). The optimisation process used 16 design parameters with efficiency as the objective function. Two different optimisers have been used, MAM and adaptive range multi-objective genetic algorithm (ARMOGA) [28]. The whole optimisation routine is presented in Fig. 14.

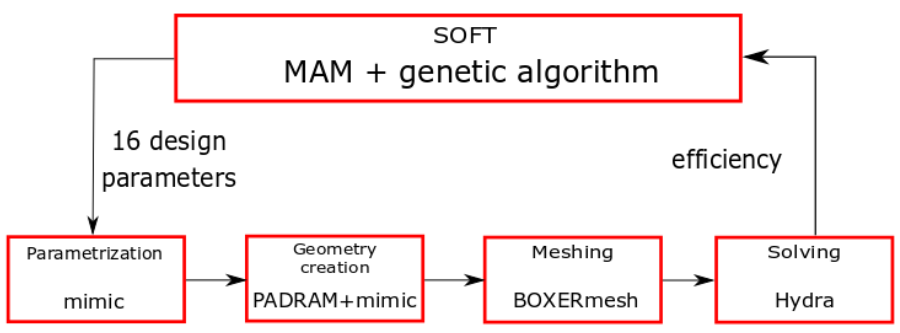

FIGURE 14. WINGLET-SQUEALER OPTIMISATION ROUTINE

MAM, which proved to quickly converge with a low complexity RBF surface (single rim squealer) was tested first on this case. However, in this optimisation, because of the general design space, MAM was not able to approximate the objective function as it moved through smaller trust regions and find an improved design. Therefore, ARMOGA was used, with and without surrogate models. To create surrogate models, the optimisation was initialized by design of experiments (DOE) with 200 cases and the results interpolated with a Kriging response surface. However, because of the high flexibility of the design space, the Kriging response surface was found to not be accurate and ARMOGA without surrogate model was used to search for improved design.

Some of the most efficient design of all simulations ran are presented in the Fig. 15. As shown, using the RBF surface de-
TABLE 2. WINGLET-TOPOLOGY FREE SQUEALER EFFICIENCIES

\begin{tabular}{cc}
\hline Case & Efficiency $(\%)$ \\
\hline a) & +0.04 \\
b) & +0.12 \\
c) & +0.13 \\
d) & +0.13 \\
e) & +0.18 \\
f) & +0.32 \\
\hline
\end{tabular}

fined by 16 points allowed different squealer topologies to be created with up to 3 parallel squealer line in pitch-wise and axialwise direction. All of the designs presented in the Fig. 15 feature minimum 2 squealer lines with opening at the LE and TE which is in agreement with findings from Schabowski and Hodson [8] and Caloni et al. [11].

The efficiency benefits compared to the flat winglet tip (which was used as a baseline tip and for designs presented in Fig. 15) are summarised in Tab. 2.

\section{OPTIMISED DESIGNS ANALYSES}

\subsection{Winglet}

Fig. 16 presents slices of relative total pressure for the datum a) and optimised winglet b) blades. Tip leakage flow for both tips forms a vortex at the blade SS which stretches downstream of the blade and is visible as a region of low total pressure. The OTL vortex starts forming at around $20 \%$ of axial chord length and for the datum flat tip forms right next to the SS of the blade, but for the winglet blade is pushed away from the blade into the region of higher pressure. Part of the flow leaking from the datum flat tip is not entrained in the OTL vortex and it drops below it causing an additional low pressure region below OTL vortex core. The OTL vortex core produced by the flat tip blade is of lower total pressure and it is stronger than the one by winglet tip, leading to increased dissipation. As the OTL vortex passes the blade, in the case of the winglet tip it is completely separated from the TE wake decreasing the shear and viscous effect. In the case of datum blade it is merged with TE wake additionally enhancing the mixing process and entropy production. These results are in agreement with the study of Coull et al. [9]. Absence of the PS overhang was studied and it was found that winglet with PS overhang has slightly lower efficiency than optimised winglet design. The reason for this is that large PS overhang causes vortex around LE-PS region which leads to an increased dissipation in the PS region close to the blade tip. Essentially, the winglet tip 


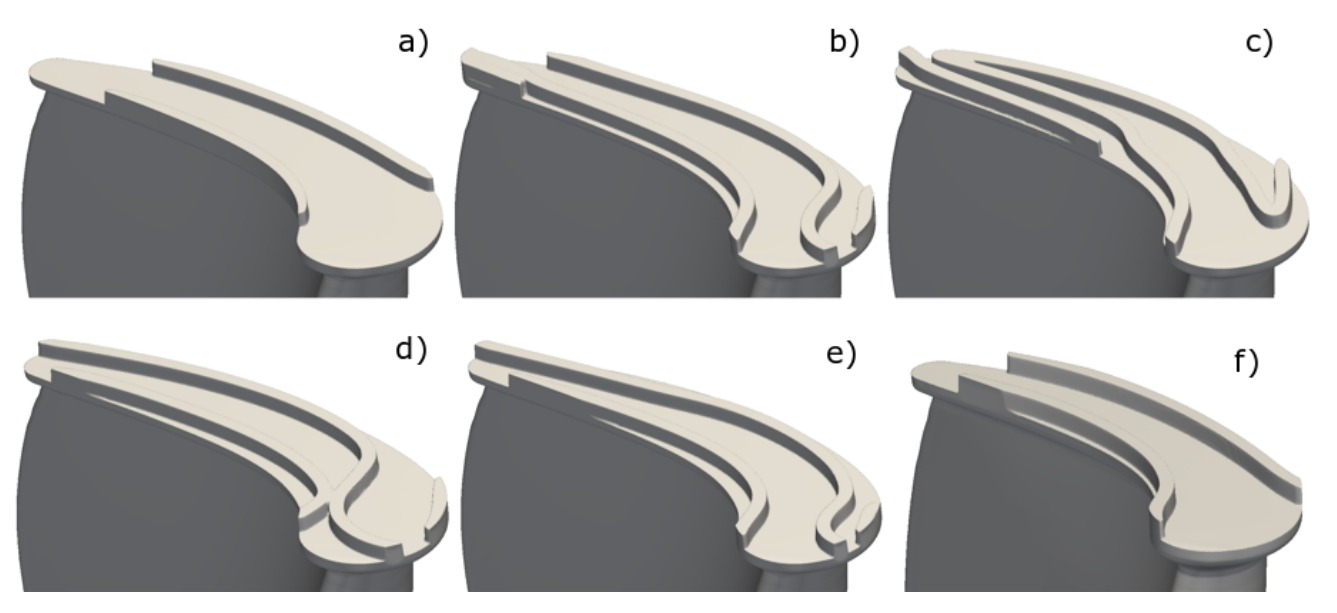

FIGURE 15. WINGLET-TOPOLOGY FREE SQUEALER DESIGNS

increases efficiency by $1.40 \%$ by moving the OTL vortex away from the blade, causing it not to interact with the blade wake.

\subsection{Single rim squealer}

Fig. 16c presents slices of relative total pressure for optimised single rim squealer. As the squealer rim is located on the PS, OTL flow separates flowing over the squealer rim, forming a vortex at the tip of the blade presented in detail of Fig. 16c. This vortex acts as a seal and is stronger at the tip region closer to the LE. Its size depends on the tip width-to-squealer wall height ratio and as the tip region gets narrower toward TE, the vortex has no space to fully form. It was found that in this case, the vortex forms in the region where tip width-to-squealer wall height ratio is greater than 2. Towards the TE, as this ratio decreases, the vortex can not fully form, which results in weakening of the sealing effect and accordingly, increase of $\dot{m}_{\text {leak }}$. Overall, optimised design increased aerodynamic efficiency by $0.46 \%$ compared to the datum flat tip blade.

Adding a single rim on the datum blade significantly changes the behaviour of the OTL vortex (compared to the datum blade, a) in Fig. 16). OTL vortex forming from the tip of the single rim squealer is divided into two vortices. One vortex is formed from the flow leaking over the partially open LE (A in Fig. 16c) and is of smaller area. Second part of OTL vortex (B in Fig. 16c) is formed by flow leaking over the squealer rim, consisting of the majority of leakage flow, therefore of greater area and lower pressure core. These two vortices are counter rotating and interact in the region after the TE.

To identify benefits of the optimised tip, designs with squealer rim placed in the middle of the tip along the camber line and at the blade SS were also examined. It was found that placing a squealer rim at the SS worsens the performance, having lower efficiency than the flat tip datum blade. The reason for this was found to be absence of sealing vortex on the blade tip. Efficiency benefit of the squealer with single rim along the camber line was found to be around half of the optimised design. Part of the flow leaking over the PS before the squealer rim was found to cause no sealing effect, behaving similar to the case of the squealer with rim on the SS. The efficiency benefit comes from the vortex which forms after the squealer rim, between the rim and the SS edge. But since this vortex has no room to fully form, is much weaker when compared to the optimised design one, causing less sealing.

\subsection{Winglet-topology free squealer}

In this section, the most efficient design will be presented (shown in the Fig.15f). The Fig. 17 presents the static pressure on the optimised blade tip expressed as isentropic Mach number. The large opening on LE causes a subsonic region in the first part of the tip, entraining the flow around LE. Subsonic region stretches along the squealer wall on the SS until $70 \%$ of the camber line, allowing a pair of tip sealing vortices to form.

Reason for forming the strong sealing region is visualised in the Fig. 18 with streamlines and slices of relative total pressure. As mentioned before, LE opening entrains the flow around the LE from where it is divided into two parts. One part flows before and over the early SS squealer wall, forming part of the OTL vortex on the blade SS. The other part of the flow entrained at the LE forms a vortex inside the tip cavity, along the PS squealer wall (visualised with streamlines). This vortex is visible as the region A of lower total pressure in the detail of the Fig. 18 and acts as a blockage. OTL flow passing over the PS squealer wall and over the vortex shown as A forms a counter rotating vortex inside the tip cavity acting as a seal (shown as B region in the detail of the Fig. 18). This vortex (B) stretches alongside SS squealer wall until around $70 \%$ of camber line and causes low pressure region along the SS on the blade tip (visible in the Fig. 17) causing strong sealing effect. 


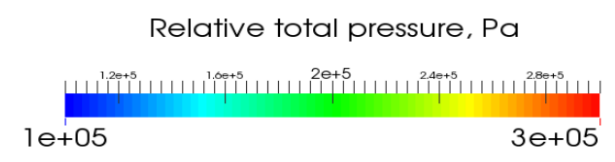

a)

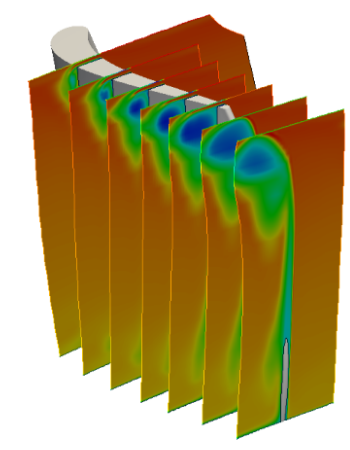

b)

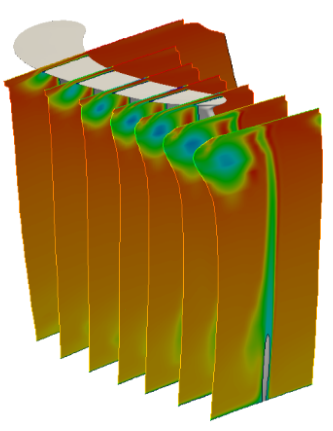

c)

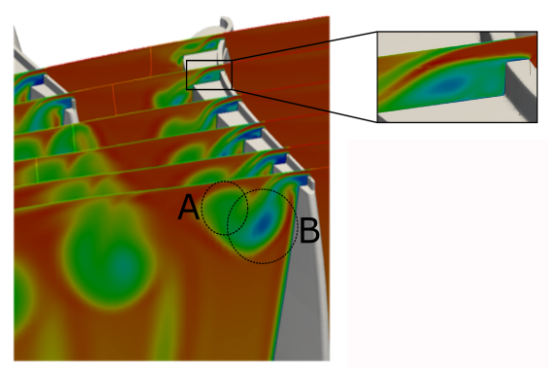

FIGURE 16. RELATIVE TOTAL PRESSURE CONTOURS FOR DATUM a), OPTIMISED WINGLET TIP b) AND SINGLE RIM SQUEALER c) BLADES

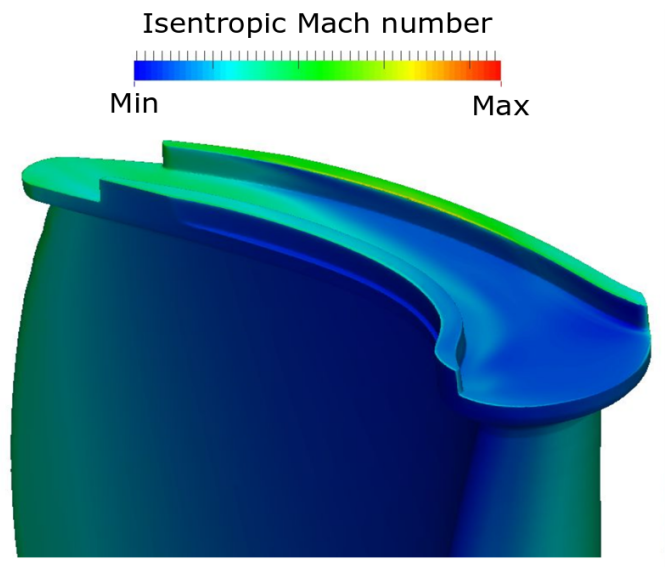

FIGURE 17. ISENTROPIC MACH NUMBER FOR OPTIMISED WINGLET-SQUEALER

As the gap between two squealer walls narrows towards the TE, B vortex does not have room to form. This causes less sealing effect (Fig. 17) and vortex shown in detail $\mathrm{C}$ in the Fig. 18 fills the whole cavity. This vortex mitigates the OTL flow by decreasing the effective tip gap, as shown in the detail C. However, this OTL prevention method is less effective than the pair of two counter rotating vortices.

\section{CONCLUSION}

This paper deals with exploring the topology optimisation of high pressure turbine blade tips. The routine implemented con-

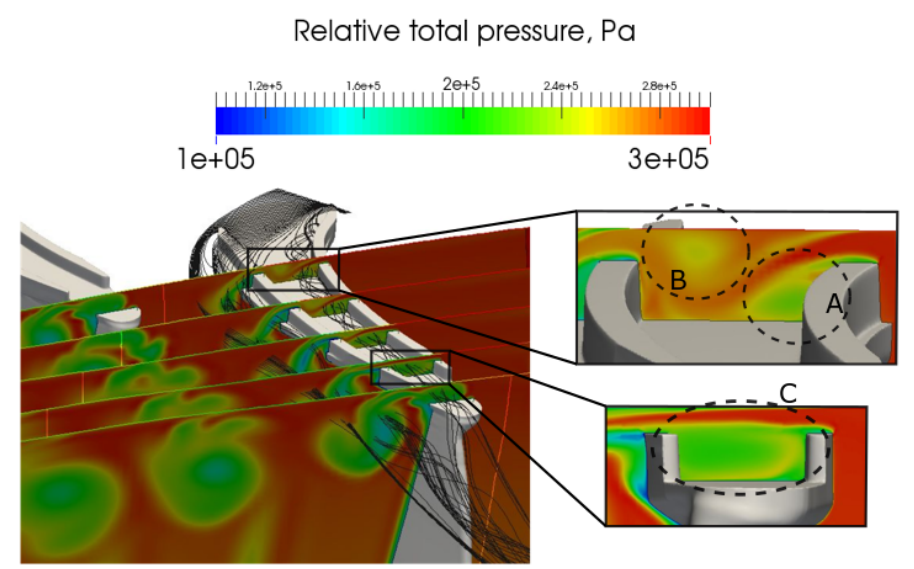

FIGURE 18. RELATIVE TOTAL PRESSURE CONTOURS FOR OPTIMISED WINLGET-SQUEALER

sists of geometry parametrization and creation, robust meshing and design assessment with RANS simulations. Novel tip shapes are presented, combining winglet and squealer based topologies.

In winglet only optimisation, it was found that the most efficient design has maximum allowed overhangs along the whole tip edge apart from the mid-PS and TE SS. Efficiency benefit was $1.40 \%$ compared to the flat tip datum blade.

During the optimisation of squealer tip with one squealer wall it was found that the most efficient design features a wall at the PS of the blade, improving efficiency by $0.46 \%$ compared to the flat tip.

Topology optimisation of novel winglet-squealer tips was found to be able to produce novel designs of different topologies 
and improved performance. The most efficient design outperforms the flat tip winglet blade and winglet with full perimeter squealer by $0.32 \%$ and $0.17 \%$ respectively.

Convergence of the optimizations was found to be directly related to the degree of freedom of the design space. Winglet and one line squealer optimisations resulted in quick convergence using MAM optimisation. However, in the topology free optimisation, because of discontinuity of the design space, optimisers that use surrogate models were found to be inadequate. Therefore, to properly optimise different tip topologies, design space needs to be subdivided using smart constrains allowing continuity but keeping capability of creating different topologies. With establishing powerful topology optimisation routine, novel designs can be further dissected using parametric study. This needs to be further investigated.

\section{ACKNOWLEDGMENTS}

Authors would like to thank Rolls-Royce plc for technical support and permission to publish this work and to Optimad Engineering for providing and support with their software used in this project. This project has received funding from the European Union's Horizon 2020 research and innovation programme under grant agreement No 769025 .

\section{REFERENCES}

[1] Denton, J. D., 1993. "Loss mechanisms in turbomachines". Journal of Turbomachinery, 115(4), October, pp. 621-656.

[2] Sieverding, C. H., 1985. "Recent progress in the understanding of basic aspects of secondary flows in turbine blade passages". Journal of Engineering for Gas Turbines and Power, 107(2), October, pp. 248-257.

[3] Coull, J. D., and Atkins, N. R., 2015. "The influence of boundary conditions on tip leakage flow". Journal of Turbomachinery, 137(6).

[4] Vass, P., and Arts, T., 2011. "Numerical investigation of high-pressure turbine blade tip flows: analysis of aerodynamics". Proceedings of the Institution of Mechanical Engineers, Part A: Journal of Power and Energy, 225(7), pp. 940-953.

[5] Pátý, M., Cernat, B. C., Maesschalck, C. D., and Lavagnoli, S., 2019. "Experimental and numerical investigation of optimized blade tip shapes-part ii:tip flow analysis and loss mechanisms". Journal of Turbomachinery, 141(11).

[6] Li, J., Du, K., and Song, L., 2016. "Effects of tip cavity geometries on the aerothermal performance of the transonic turbine blade with cavity tip". Proceedings of the Institution of Mechanical Engineers, Part A: Journal of Power and Energy, 230(3), pp. 319-331.

[7] Du, K., Li, Z., Li, J., and Sunden, B., 2019. "Influences of a multi-cavity tip on the blade tip and the over tip cas- ing aerothermal performance in a high pressure turbine cascade". Applied Thermal Engineering, 147, pp. 347-360.

[8] Schabowski, Z., and Hodson, H., 2014. "The reduction of over tip leakage loss in unshrouded axial turbines using winglets and squealers". Journal of Turbomachinery, 136(4), April.

[9] Coull, J. D., Atkins, N. R., and Hodson, H. P., 2014. "Winglets for improved aerothermal performance of high pressure turbines". Journal of Turbomachinery, 136(9).

[10] Coull, J. D., Atkins, N. R., and Hodson, H. P., 2014. "High Efficiency Cavity Winglets for High Pressure Turbines". Vol. 2C: Turbomachinery of Turbo Expo: Power for Land, Sea, and Air.

[11] Caloni, S., Shahpar, S., and Coull, J. D., 2016. "Numerical investigations of different tip designs for shroudless turbine blades". Proceedings of the Institution of Mechanical Engineers, Part A: Journal of Power and Energy, 230(7), pp. 709-720.

[12] Maesschalck, C. D., Lavagnoli, S., and Paniagua, G., 2014. "Blade tip shape optimization for enhanced turbine aerothermal performance". Journal of Turbomachinery, 136(4).

[13] Maesschalck, C. D., Lavagnoli, S., Paniagua, G., Verstraete, T., Olive, R., and Picot, P., 2016. "Heterogeneous optimization strategies for carved and squealer-like turbine blade tips". Journal of Turbomachinery, 138(12).

[14] Andreoli, V., Braun, J., Paniagua, G., De Maesschalck, C., Bloxham, M., Cummings, W., and Langford, L., 2019. "Aerothermal Optimization of Fully Cooled Turbine Blade Tips". Journal of Turbomachinery, 141(6).

[15] Milli, A., and Shahpar, S., 2012. "PADRAM: Parametric Design and Rapid Meshing System for Complex Turbomachinery Configurations". Vol. Volume 8: Turbomachinery, Parts A, B, and C of Turbo Expo: Power for Land, Sea, and Air, pp. 2135-2148.

[16] mimic - Computer Aided surface manipulation and mesh morphing, http://www.optimad.it/products / mimic/, Accessed: 2019-11-15.

[17] OPTIMAD, http://www.optimad.it/, Accessed: 2019-01-27.

[18] Beard, P. F., Povey, T., and Chana, K. S., 2009. “Turbine Efficiency Measurement System for the QinetiQ Turbine Test Facility". Journal of Turbomachinery, 132(1).

[19] Lapworth, L., and Shahpar, S., 2004. "Design of gas turbine engines using CFD". In Proceedings of the ECCOMAS, pp. 24-28.

[20] Demargne, A. A., Evans, R., Tiller, P., and Dawes, W. N. Practical and Reliable Mesh Generation for Complex, Real-World Geometries.

[21] Beard, P. F., Smith, A. D., and Povey, T., 2011. "Experimental and computational fluid dynamics investigation of the efficiency of an unshrouded transonic high pressure tur- 
bine". Proceedings of the Institution of Mechanical Engineers, Part A: Journal of Power and Energy, 225(8), pp. 1166-1179.

[22] Salvadori, S., Montomoli, F., Martelli, F., Adami, P., Chana, K. S., and Castillon, L., 2011. "Aerothermal Study of the Unsteady Flow Field in a Transonic Gas Turbine With Inlet Temperature Distortions". Journal of Turbomachinery, 133(3). 031030.

[23] Salvadori, S., Montomoli, F., Martelli, F., Chana, K. S., Qureshi, I., and Povey, T., 2011. "Analysis on the Effect of a Nonuniform Inlet Profile on Heat Transfer and Fluid Flow in Turbine Stages". Journal of Turbomachinery, 134(1), 05. 011012.

[24] Shahpar, S., 2005. "SOPHY: An integrated CFD based automatic design optimisation system". ROLLS ROYCE PLCREPORT-PNR, 93022.

[25] Polynkin, A., Toropov, V., and Shahpar, S., 2008. "Adaptive and parallel capabilities in the multipoint approximation method". In AIAA/ISSMO Multidisciplinary Analysis and Optimization Conference, no. AIAA-2 in Conference Proceeding Series, AIAA.

[26] Caloni, S., Shahpar, S., and Toropov, V., 2018. "Multidisciplinary design optimisation of the cooled squealer tip for high pressure turbines". Aerospace, 5(4), p. 116.

[27] Shahpar, S., 2002. "SOFT : A New Design and Optimisation Tool for Turbomachinery". Evolutionary Methods for Design, Optimisation and Control, E.d.: Ginnakoglou, K., et.al., CIMNE.

[28] Obayashi, S., and Sasaki, D., 2004. "Multi-objective optimization for aerodynamic designs by using armogas". In Proceedings. Seventh International Conference on High Performance Computing and Grid in Asia Pacific Region, 2004., pp. 396-403. 